\title{
Hierarchical Lignin-Based Carbon Matrix and Carbon Dot Composite Electrodes for High-Performance Supercapacitors
}

Lu Yu ${ }^{1,2}$, Chien-Te Hsieh ${ }^{3,4, *}$, David J. Keffer ${ }^{2}$, Hao Chen ${ }^{5}$, Gabriel A. Goenaga ${ }^{7}$, Sheng Dai ${ }^{5,6}$, Thomas A. Zawodzinski ${ }^{7,8}$, David P. Harper ${ }^{1,2, *}$

1 Center for Renewable Carbon, Institute of Agriculture, The University of Tennessee, Knoxville, Tennessee 37996, United States

2 Department of Materials Science and Engineering, The University of Tennessee, Knoxville, Knoxville, Tennessee 37996, United States

3 Department of Chemical Engineering and Materials Science, Yuan Ze University, Taoyuan 32003, Taiwan

4 Department of Mechanical, Aerospace, and Biomedical Engineering, The University of Tennessee, Knoxville, TN 37996, United States

5 Department of Chemistry, The University of Tennessee, Knoxville, TN 37996, United States

6 Chemical Sciences Division, Oak Ridge National Laboratory, Oak Ridge, TN 37831, United States

7 Department of Chemical and Biomolecular, The University of Tennessee, Knoxville, TN 37996, United States

8 Physical Chemistry of Materials Group, Oak Ridge National Laboratory, Oak Ridge, TN 37831, United States

* Corresponding authors' email:

dharper4@utk.edu (Prof. D.P. Harper) and cthsieh@saturn.yzu.edu.tw (Prof. C.T. Hsieh) 
Table S1. Pore characteristics of AC, CD@AC-11 samples determined from $\mathrm{N}_{2}$ physisorption at $-196^{\circ} \mathrm{C}$.

\begin{tabular}{ccccc}
\hline $\begin{array}{c}\text { Carbon } \\
\text { sample }\end{array}$ & $\begin{array}{c}S_{\mathrm{BET}}{ }^{1} \\
\left(\mathrm{~m}^{2} \mathrm{~g}^{-1}\right)\end{array}$ & $\begin{array}{c}V_{\mathrm{T}}{ }^{2} \\
\left(\mathrm{~cm}^{3} \mathrm{~g}^{-1}\right)\end{array}$ & $\begin{array}{r}X_{\text {micro }}{ }^{3} \\
(\%)\end{array}$ & $\begin{array}{c}X_{\text {meso }}{ }^{4} \\
(\%)\end{array}$ \\
\hline $\mathrm{AC}$ & 591.7 & 0.34 & 77.1 & 22.9 \\
$\mathrm{CD} @ \mathrm{AC}-11$ & 299.0 & 0.11 & 67.7 & 32.3 \\
\hline
\end{tabular}

\section{Remark:}

${ }^{1} S_{\mathrm{BET}}: \quad$ Surface area determined from Brunauer-Emmett-Teller (BET) equation.

${ }^{2} V_{\mathrm{T}}: \quad$ Total pore volume determined from single-point adsorption volume at $\mathrm{p} / \mathrm{p}_{0} \sim 0.98$.

${ }^{3} X_{\text {meso }}$ : Mesopore fraction determined from the ratio of $S_{\mathrm{BET}}$ to Barrett-Joyner-Halenda (BJH) surface area.

${ }^{4} X_{\text {micro }}: \quad$ Micropore fraction determined by subtracting BJH surface area from $S_{\text {BET }}$. 
Table S2. Summary of various C-QD based EDLC electrode materials in literatures.

\begin{tabular}{|c|c|c|c|c|c|c|}
\hline Material & $\begin{array}{c}\mathrm{SSA} \\
\left(\mathrm{m}^{2} \mathrm{~g}^{-1}\right)\end{array}$ & Capacity & $\begin{array}{l}\text { Current } \\
\text { density }\end{array}$ & $\begin{array}{l}\text { Power } \\
\text { density }\end{array}$ & $\begin{array}{l}\text { Energy } \\
\text { density }\end{array}$ & Reference \\
\hline CDs/graphene ${ }^{1}$ & 435.1 & $\begin{array}{l}91.9 \\
\mathrm{~F} \mathrm{~g}^{-1}\end{array}$ & $\begin{array}{c}0.1 \\
\mathrm{~A} \mathrm{~cm}^{-2}\end{array}$ & $\begin{array}{c}15 \\
\mathrm{~mW} \mathrm{~cm} \mathrm{~cm}^{-2}\end{array}$ & $\begin{array}{c}46.67 \\
\mu \mathrm{Wh} \mathrm{cm}^{-2}\end{array}$ & No. S1 \\
\hline $\begin{array}{l}\text { GQDs/carbon fiber/ } \\
\text { graphene hydrogel }^{2}\end{array}$ & 13.48 & $\begin{array}{c}93.7 \\
\mathrm{~F} \mathrm{~cm}^{-3}\end{array}$ & $\begin{array}{c}0.2 \\
\mathrm{~mA} \mathrm{~cm}\end{array}$ & $\begin{array}{c}200 \\
\mathrm{~W} \mathrm{~kg}^{-1}\end{array}$ & $\begin{array}{l}20.5 \\
\mathrm{Wh} \mathrm{kg}^{-1}\end{array}$ & No. S2 \\
\hline CQD/Denatured milk ${ }^{3}$ & 1 & $\begin{array}{l}95.0 \\
\mathrm{~F} \mathrm{~g}^{-1}\end{array}$ & $\begin{array}{l}0.12 \\
\mathrm{~A} \mathrm{~g}^{-1}\end{array}$ & 1 & 1 & No. S3 \\
\hline C(ZIF-8)@GQDs ${ }^{4}$ & 668 & $\begin{array}{c}130 \\
\mathrm{~F} \mathrm{~g}^{-1}\end{array}$ & $\begin{array}{c}2 \\
\mathrm{~A} \mathrm{~g}^{-1}\end{array}$ & 1 & 1 & No. S4 \\
\hline GQDs/graphene oxide 5 & 214.0 & $\begin{array}{c}242 \\
\mathrm{~F} \mathrm{~g}^{-1}\end{array}$ & $\begin{array}{l}1.17 \\
\mathrm{~A} \mathrm{~g}^{-1}\end{array}$ & 1 & 1 & No. S5 \\
\hline $\begin{array}{c}\text { GQDs/ultramicroporous } \\
\text { carbons }^{6}\end{array}$ & 1730.0 & $\begin{array}{l}270 \\
\mathrm{~F} \mathrm{~g}^{-1}\end{array}$ & $\begin{array}{c}1 \\
\mathrm{~A} \mathrm{~g}^{-1}\end{array}$ & $\begin{array}{c}70 \\
\mathrm{~W} \mathrm{~kg}^{-1}\end{array}$ & $\begin{array}{c}9.38 \\
\text { Wh kg }^{-1}\end{array}$ & No. S6 \\
\hline CD@AC & 299.0 & $\begin{array}{l}301.7 \\
\mathrm{~F} \mathrm{~g}^{-1}\end{array}$ & $\begin{array}{l}0.15 \\
\mathrm{~A} \mathrm{~g}^{-1}\end{array}$ & $\begin{array}{l}12500 \\
\mathrm{~W} \mathrm{~kg}^{-1}\end{array}$ & $\begin{array}{c}11 \\
\text { Wh kg k}^{-1}\end{array}$ & This work \\
\hline ONCDs/Hydrogels ${ }^{7}$ & 1011.0 & $\begin{array}{l}483 \\
\mathrm{~F} \mathrm{~g}^{-1}\end{array}$ & $\begin{array}{c}1 \\
\mathrm{~A} \mathrm{~g}^{-1}\end{array}$ & $\begin{array}{l}11000 \\
\mathrm{~W} \mathrm{~kg}^{-1}\end{array}$ & $\begin{array}{c}13.5 \\
\text { Wh kg-1 }\end{array}$ & No. S7 \\
\hline
\end{tabular}




\section{References}

S1. Li, Q.; Cheng, H.; Wu, X.; Wang, C.-F.; Wu, G.; Chen, S., Enriched carbon dots/graphene microfibers towards high-performance micro-supercapacitors. J. Mater. Chem. A 2018, 6 (29), 14112-14119.

S2. Li, Z.; Wei, J.; Ren, J.; Wu, X.; Wang, L.; Pan, D.; Wu, M., Hierarchical construction of high-performance all-carbon flexible fiber supercapacitors with graphene hydrogel and nitrogen-doped graphene quantum dots. Carbon 2019, 154, 410-419.

S3. Athika, M.; Prasath, A.; Duraisamy, E.; Sankar Devi, V.; Selva Sharma, A.; Elumalai, P., Carbon-quantum dots derived from denatured milk for efficient chromium-ion sensing and supercapacitor applications. Mater. Lett. 2019, 241, 156-159.

S4. Yu, H.; Zhu, W.; Zhou, H.; Liu, J.; Yang, Z.; Hu, X.; Yuan, A., Porous carbon derived from metal-organic framework@graphene quantum dots as electrode materials for supercapacitors and lithium-ion batteries. RSC Adv. 2019, 9 (17), 9577-9583.

S5. Luo, P.; Guan, X.; Yu, Y.; Li, X.; Yan, F., Hydrothermal Synthesis of Graphene Quantum Dots Supported on Three-Dimensional Graphene for Supercapacitors. Nanomaterials (Basel) 2019, 9 (2).

S6. Zhang, S.; Zhu, J.; Qing, Y.; Wang, L.; Zhao, J.; Li, J.; Tian, W.; Jia, D.; Fan, Z., Ultramicroporous Carbons Puzzled by Graphene Quantum Dots: Integrated High Gravimetric, Volumetric, and Areal Capacitances for Supercapacitors. Adv. Funct. Mater. 2018, 28 (52).

S7. Wei, J.-S.; Song, T.-B.; Zhang, P.; Zhu, Z.-Y.; Dong, X.-Y.; Niu, X.-Q.; Xiong, H.-M., Integrating Carbon Dots with Porous Hydrogels to Produce Full Carbon Electrodes for Electric Double-Layer Capacitors. ACS Appl. Energy Mater. 2020, 3 (7), 6907-6914. 